\title{
Endoscopic submucosal dissection with a novel high viscosity injection solution (LiftUp) in an ex vivo model: a prospective randomized study
}

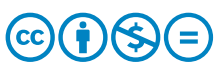

\author{
Authors \\ E. Wedi', P. Koehler ${ }^{2}$, J. Hochberger ${ }^{3}$, J. Maiss ${ }^{4}$, S. Milenovic ${ }^{1}$, M. Gromski ${ }^{5}$, N. Ho ${ }^{6}$, C. Gabor ${ }^{6}$, U. Baulain ${ }^{2}$, \\ V. Ellenrieder ${ }^{1}$, C. Jung ${ }^{1}$
}

Institutions

1 Department of Gastroenterology and Gastrointestinal Oncology, Center of Interdisciplinary Endoscopy, University Medical Centre Göttingen, Göttingen, Germany

2 Institute of Farm Animal Genetics, Friedrich-LoefflerInstitut (FLI), Federal Research Institute for Animal Health, Mariensee, Germany

3 Department of Gastroenterology, Vivantes Klinikum in Friedrichshain, Teaching Hospital of Charité Humboldt University, Berlin, Germany

4 Department of Gastroenterology, Associates Dr. Kerzel and Prof. Maiss, Forchheim, Germany

5 Division of Gastroenterology and Hepatology, Indiana University School of Medicine, Indianapolis, IN, USA

6 Ovesco Endoscopy AG, Tübingen, Germany

submitted 5.7.2018

accepted after revision $\quad 2.11 .2018$

Bibliography

DOI https://doi.org/10.1055/a-0874-1844 |

Endoscopy International Open 2019; 07: E641-E646

(c) Georg Thieme Verlag KG Stuttgart · New York elSSN 2196-9736

Corresponding author

Edris Wedi, MD, Interdisciplinary Endoscopy, Department of Gastroenterology and GI Oncology, University Medical Centre Göttingen, Georg-August-University, D-37075

Göttingen, Germany

Fax: +49-512-1901241

edris.wedi@med.uni-goettingen.de

\section{ABSTRACT}

Introduction Endoscopic submucosal dissection (ESD) is increasingly being used in the western world. Submucosal injectates are an essential tool for the ESD procedure. In this study, we evaluated a novel copolymer injectate (LiftUp, Ovesco, Tübingen Germany) in an established ESD model (EASIE-R) in comparison to existing submucosal injectables.

Materials and methods We conducted a prospective, randomized ex vivo study performing ESD with three injectates: LiftUp, hydroxyethyl starch (HAES 6\%) and normal saline solution $(\mathrm{NaCl} 0.9 \%)$. A total of 60 artificial lesions, each $3 \times 3 \mathrm{~cm}$ in size, were resected in an ex vivo porcine model, utilizing one of the three studied injectates $(n=20$ ESDs per injectate). Study parameters were: en bloc resection rate, perforation rate, lifting property, time of injection, injectate volume, general ESD procedure time, and overall procedure time.

Results All 60 lesions were successfully resected using the standard ESD technique. LiftUp had no procedure related perforations, one perforation occurred in the HAES group, and two perforations in the $\mathrm{NaCl}$ group $(P>0.05)$. Furthermore, adequate lifting was achieved in 16/20 (80\%) using LiftUp, 6/20 (30\%) in the HAES group and 6/20 (30\%) in the $\mathrm{NaCl}$ group $(P<0.0002)$. En bloc resection was achieved in 19 (95\%) with LiftUp, in 20 (100\%) with HAES, and in 16 (80\%) with $\mathrm{NaCl}$. General ESD procedure time and overall procedure time were not different among the three groups. Conclusion LiftUp appears to be a safe alternative to established fluids for ESD. It had a significantly improved lifting effect and required significantly less injected volume compared to well-established lifting solutions.

\section{Introduction}

Endoscopic mucosal resection (EMR) and endoscopic submucosal dissection (ESD) are established techniques for the treatment of precancerous lesions and early stage cancer in the western world $[1,2]$. The ESD technique was originally developed for the resection of gastric neoplasms, but is increasingly used in the colon and the esophagus. Compared to EMR, ESD has certain advantages, including a higher rate of en bloc resection with a precise histopathological examination. In addition, resection of larger $(>2 \mathrm{~cm})$ lesions is possible with simultaneously lower recurrence rates [3-5]. On the other hand, ESD is an ad- 
vanced technique with a long learning curve, even for experienced endoscopists, has a longer procedure time than EMR and a higher incidence of adverse events. One of the major complications is perforations, with an incidence up to $4-10 \%$ [2]. For that reason, perforation prevention is essential, and the most effective way to do this is to use an adequate and effective submucosal fluid cushion [6]. For this reason, the ESGE guidelines specify an ideal submucosal injection fluid which provides a sustainable lift, facile en bloc resection, low cost, widespread availability and few adverse events [7].

To date, the most studied injection fluid is $\mathrm{NaCl} 0.9 \%$, beginning in the early 1950s [8], and with the advantages of widespread availability and low cost $[9,10]$. The limitation of $\mathrm{NaCl}$ $0.9 \%$ is that it does not provide a long lasting cushion effect and repeated injections are necessary even after a short period of time, which prolongs the procedure time [11,12].

Other solutions studied include gelatin, hydroxypropylmethylcellulose (HPMC), hydroxyethyl starch (HAES) (Hetastarch, Hespan), hyaluronic acid, glucose, dextrose, fructose, glycerine, Na-alginate, Eleview (Aries Pharmaceuticals, San Diego, California, United States) and mixtures of all those components $[9,13]$. Up to now, no clear recommendation exists to support the use of one fluid over another; however, comments have been made in position and guideline papers (ESGE) promoting the use of more viscous substances over normal saline in EMR $[7,14]$. There is an absolute necessity for research in this field.

It is for this reason that a new highly viscous injectate solution (LiftUp, Ovesco Endoscopy AG, Tübingen, Germany) was developed. LiftUp is composed of distilled water, and a co-polymer derived from ethylene oxide, propylene oxide and sodium chloride, and has a thermo-gelling behavior. It is a co-polymer, which is fluid at low temperatures, and forming micelles above a critical temperature. The micelles connect above the critical micelle temperature and form a grid, which increases the viscosity of the fluid. Maximum viscosity is reached at body temperature $\left(37^{\circ} \mathrm{C}\right)$. This is a reversible effect, which allows for washing out LiftUp by cooling the resection site with cold water. It builds a gelatinous cushion, which remains stable and therefore enables subsequent resection without reinjection.

The aim of this study is to evaluate different parameters of the ESD procedure with the thermo-sensitive injectate LiftUp in comparison to $\mathrm{NaCl} 0.9 \%$ and $\mathrm{HAES} 6 \%$ (control group) in an established ex vivo ESD model.

\section{Materials and methods}

This study was exempt from IRB review since no animal or human subjects were included. It was designed as a prospective, randomized ex vivo study. The experiments were carried out at the Federal Research Institute for Animal Health in Mariensee, Germany. Stomachs from pigs (German Landrace, live weight $60 \mathrm{~kg}$ ) were cleaned and frozen after slaughtering. The stomachs were thawed before the procedure and placed in the EASIE-R simulator ( $>$ Fig. 1). This model has previously been evaluated in different endoscopic procedures and is an established model for interventional endoscopic training and research [15, 16].

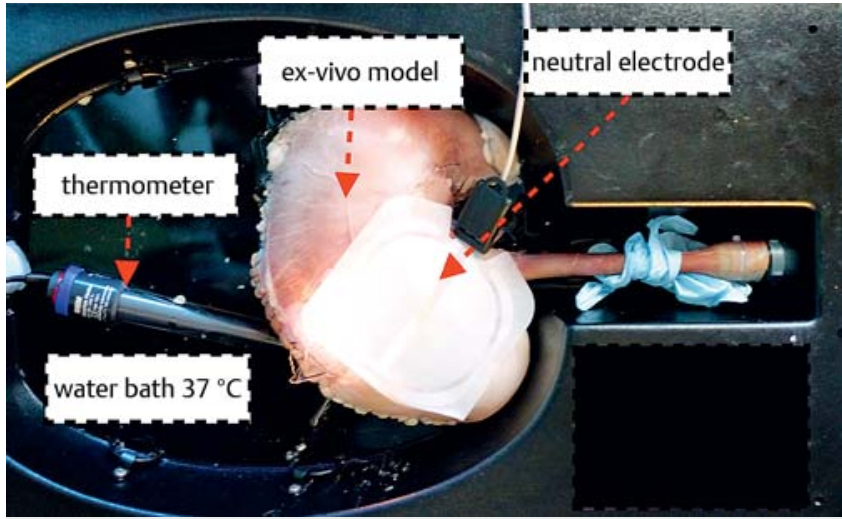

- Fig. 1 EASIE-R simulator for ESD procedures. The porcine stomach is thawed before the procedure and placed in the EASIE-R simulator. A water bath at $37^{\circ} \mathrm{C}$ was used to simulate the in vivo environment and keep the porcine stomach homothermal.

All 60 procedures were performed by one endoscopist, who had previous experience in performing gastric ESD in the ex vivo simulator (>60 ESDs before this study) and was blinded to the injection fluid used.

LiftUp was compared to two standard submucosal injection fluids: hydroxyethyl starch (HAES 6\%, B. Braun, Melsungen, Germany) and normal saline ( $\mathrm{NaCl} 0.9 \%$, B. Braun, Melsungen, Germany). In total, 20 ESD procedures were performed for each injection fluid.

\section{Preparation of LiftUp for submucosal injection}

At low temperatures, LiftUp is liquid and forms a gel-like consistency with increasing temperature. Due to its higher viscosity, an inflation device (Accura Medizintechnik $\mathrm{GmbH}$, Ref. no. $8610230 S$ ) was used to deliver the material through the injection needle (needle length $6 \mathrm{~mm}$, needle diameter $0.7 \mathrm{~mm}$ ), providing a pressure of up to 20 bars. To keep the conditions comparable, the same injection needle was also used for HAES and $\mathrm{NaCl}$ injections. After submucosal injection of LiftUp, the material was then left in place in the submucosa for approximately $1-3$ minutes to form the desired gel-like configuration. A water bath at $37^{\circ} \mathrm{C}$ was used to simulate the in vivo environment and keep the porcine stomach homothermal ( $\triangleright$ Fig.1). After 5 minutes, endoscopic resection was initiated. Lifting properties were measured by the blinded researcher using a subjective score from 1 to 5 points, with 5 points being the best valuation and representing properties of an ideal lifting injection with an efficient submucosal cushion. Ovesco is currently in the process of applying for CE marking for LiftUp.

\section{ESD procedure}

Before the interventions, standardized lesions, each measuring $3 \times 3 \mathrm{~cm}$, were manually placed in the corpus of the porcine stomach ( $\triangleright$ Fig.2). The stomach was placed in the EASIE-R model, which consists of a plastic hull, where the esophagus and the pig stomach are secured [15]. Six ESD lesions were created per ex vivo stomach. The endoscopist was blinded with regard to which injection solution was provided for the resection. 


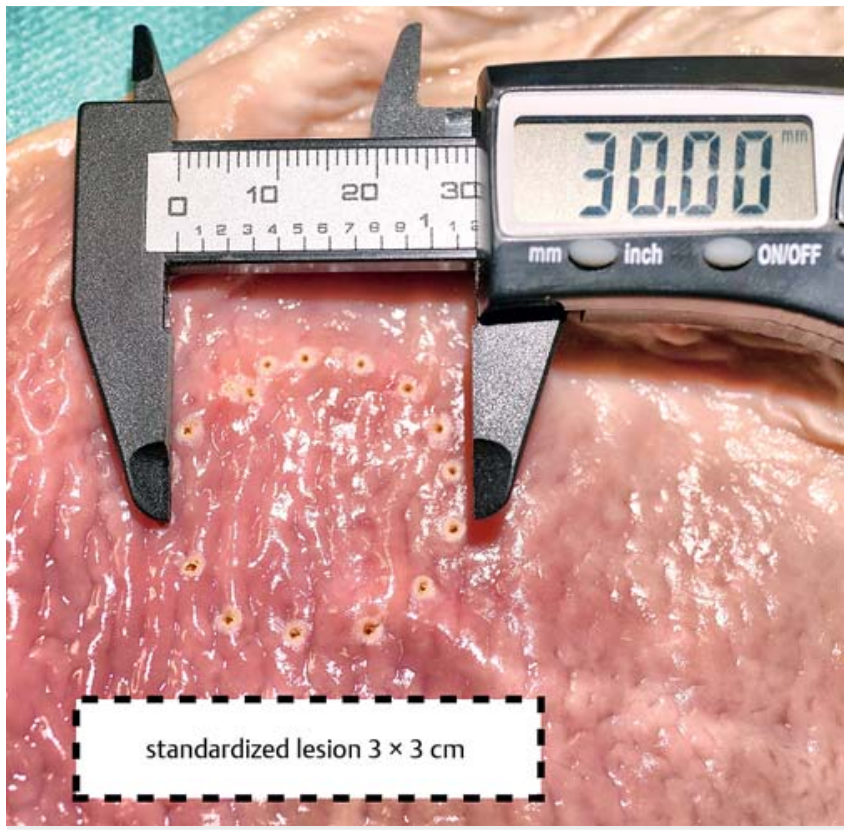

- Fig. 2 Standardized lesions to be resected by ESD were manually placed in the corpus of porcine stomach, each measuring $3 \times 3 \mathrm{~cm}$.

For randomization, 60 identical lots (20 for each injection solution) were drawn from a pot. Before injection, the LiftUp solution was stored cooled between $2{ }^{\circ} \mathrm{C}$ and $8{ }^{\circ} \mathrm{C}$ in a refrigerator. All lesions were resected using the classic ESD technique. The procedures were performed sequentially, over a study period of 1 month. ESD was performed using a gastroscope (EG-530 D Fujinon, Fujifilm, Japan), an ESD cap (DH 29CR ST, Hood short type, Fujifilm, Japan) and a FlushKnife $1.5 \mathrm{~mm}$ (Fujinon, Fujifilm, Japan). Injection fluids were mixed with Methylene Blue dye (Amino AG, Gebenstorf, Switzerland) to optimize visualization and differentiation of submucosal fibers during the ESD. The electrosurgical unit used was the ERBE VIAO 200 (ERBE Elektromedizin, Tübingen, Germany). For the ESD, the settings on the ERBE device were EndoCut Q 1/1/1.

\section{Data collection}

An independent observer recorded the following parameters: time required for fluid injection, amount of fluid injected, time required for circumferential incision, ESD procedure time, overall procedure time (submucosal dissection time), en bloc resection rate, and perforation rate. Data concerning all parameters mentioned were collected during each ESD. The resected specimens were spread and pinned on cork plates after each ESD procedure and photo documented ( $>$ Fig. 3 ). The ESD procedure time was defined as the time from the beginning of the ESD procedure, after circumferential incision, to complete resection of the lesion from the porcine stomach. Each resection site was evaluated visually from the endoscopic site in addition to an insufflation test to determine the presence of a perforation.
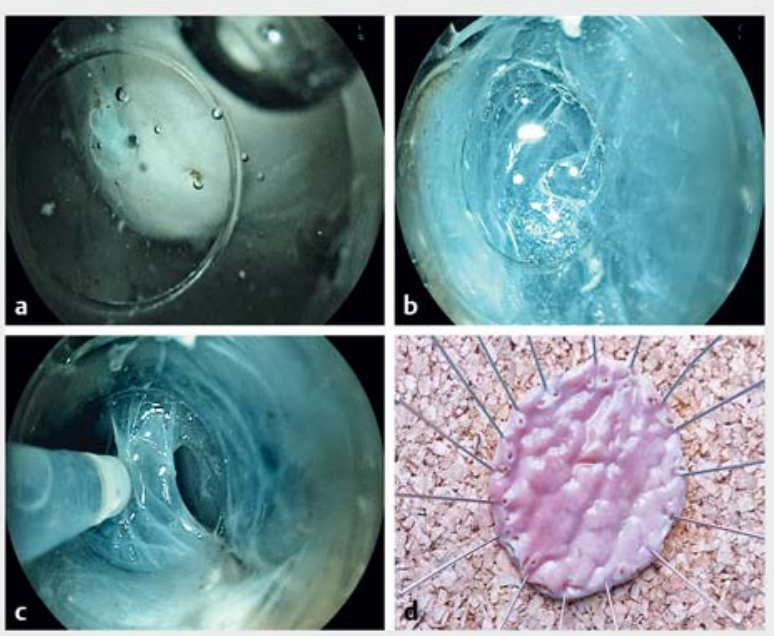

- Fig. 3 Steps in the ESD procedure with LiftUp. a Submucosal cushion after LiftUp injection. $\mathbf{b}$ - $\mathbf{d}$ Submucosal dissection with a FlushKnife. The submucosal cushion remains stable with LiftUp during the entire procedure. The specimen was spread and pinned on a cork mat after the ESD procedure.

\section{Statistical analysis}

Data analysis was performed using JMP 11 (SAS Institute Inc., 2014) and R 3.3.3 (R Core Team, 2017) software. Box and whisker plots were used to graphically represent the spread in the data by showing the quartiles of the data along with the extreme values. The bottom and top of the box are the 25th (Q1) and 75th (Q3) percentiles, and the band near the middle of the box is the median (50th percentile, Q2). The ends of the whiskers represent Q1 minus $1.5 \times$ interquartile range (IQR) and Q3 plus $1.5 \times I Q R$, respectively.

For continuous variables (injection time, etc.), differences between injection fluid groups were tested using a KruskalWallis test (one-way ANOVA on ranks), followed by nonparametric comparisons of each pair using the Wilcoxon method when the global test was significant. For binary variables (en bloc resection and adverse events), we used Fisher's exact test to compare the differences between injection fluid groups. A $P$ value less than 0.05 was considered to be statistically significant.

\section{Results}

\section{Injection time for the submucosal injectates}

Due to its more viscous properties, the injection time with LiftUp (median 4 minutes, range $2-7.8$ minutes) was significantly longer than HAES (median 3 minutes, range $1-6.9$ minutes, $P=0.0177$ ), and $\mathrm{NaCl}$ (median 3 minutes, range $1-6$ minutes, $P=0.025)$. 


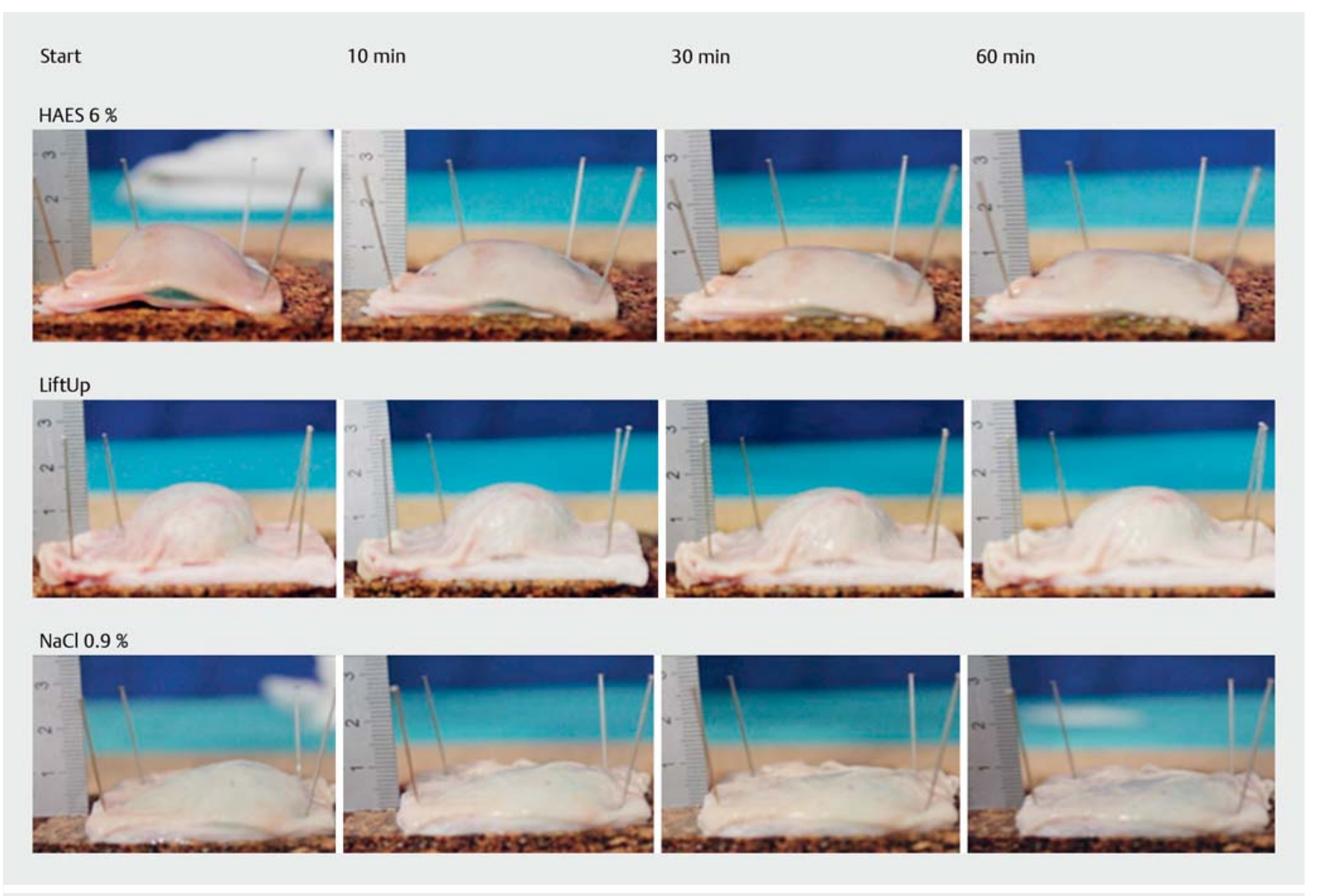

Fig. 4 Lifting persistency under simulation circumstances in the porcine model for HAES 6\%, LiftUp, and $\mathrm{NaCl} 0.9 \%$.

\section{Lifting properties and required volume of the submucosal injectates}

The lifting persistency of all three injection solutions is presented in Fig. 4. Injection with LiftUp received the best scores with 16 of 20 receiving 5 points. The median quality score for HAES $=4$ vs. median LiftUp 5, $P=0.0005$. The median quality score for $\mathrm{NaCl}=3$ vs. LiftUp 5, $P=0.0002$.

The overall injection volume needed for adequate lifting during ESD was significantly lower in the LiftUp group compared to the HAES (median $33.5 \mathrm{~mL}$ vs. $231 \mathrm{~mL}, P<0.0001$ ) or normal saline groups (median $33.5 \mathrm{~mL}$ vs. $299 \mathrm{~mL}, P<0.0001$ ) ( $>$ Fig.5). Within 60 minutes of the injection, only LiftUp permitted a stable cushion.

With regard to the time required for the circumferential cut, there was no statistically significant difference between the injection groups (LiftUp vs. HAES: median 10.5 minutes vs. 8.0 minutes, $P=0.5688 /$ LiftUp vs. $\mathrm{NaCl}$ : median 8.0 minutes vs. 7.5 minutes, $P=0.2219$ ).

The variability of the ESD procedure time was lower in the LiftUp group compared to the other two groups. However, despite a trend towards lower ESD procedure time in the LiftUp group compared to the $\mathrm{NaCl}$ group, the differences did not reach statistical significance (LiftUp vs. HAES: median 19.5 minutes vs. 22.0 minutes, $P=0.3296 /$ LiftUp vs. $\mathrm{NaCl}$ : 19.5 minutes vs. 25.0 minutes, $P=0.0544)$ ( $\triangleright$ Fig. 6 ).
Similarly, there were no statistically significant differences in total procedure time between groups.

\section{En bloc resection rate}

En bloc resection was achieved in 20 out 20 ESD cases when HAES was used, compared to 19 out of 20 ESDs in the LiftUp group and 16 out of 20 ESDs in the $\mathrm{NaCl}$ group $(P>0.05)$.

\section{Adverse events}

Due to the ex vivo nature of the ESD model, peri- or post-ESD bleeding was not able to be simulated. No perforation occurred during ESD in the LiftUp group $(n=0 / 20)$, whereas one perforation occurred in the HAES group $(1 / 20)$ and two in the normal saline group $(2 / 20)(P>0.05)$.

\section{Discussion}

The purpose of this study was to evaluate the performance characteristics of three submucosal injection materials for ESD, including a new thermosensitive co-polymer (LiftUp), in comparison to established injectates. ESD and EMR are minimally invasive endoscopic procedures increasingly accepted for treatment of dysplastic and early malignant lesions limited to the superficial layers of the gastrointestinal wall $[13,17]$. The disadvantages of EMR are a relatively higher recurrence 


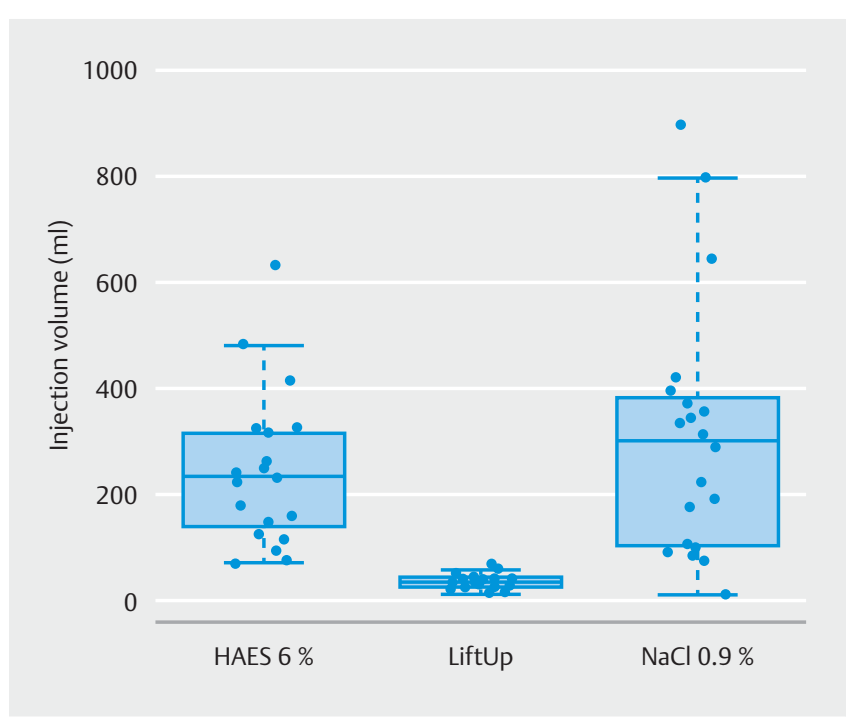

- Fig. 5 Injection volume $(\mathrm{mL})$ dependent on the injection substance $(\mathrm{mL})$.

rate, especially when piecemeal EMR is performed [18]. ESD is superior to EMR in achieving en bloc resection for large lesions [19].

The submucosal cushion, produced with the injection of a solution into the submucosal space, is an essential step in ESD with regard to ensuring the safety and efficiency of the procedure. An ideal injection substance should create a long lasting "cushion" effect by expanding the submucosal space, thereby preventing perforations and limiting the need of recurrent injections.

The ESGE guidelines have recommended more viscous injection solutions over the use of normal saline for endoscopic mucosal resections [7]. Special technical recommendations concerning injection fluids in ESD do not exist yet and most of the commonly used submucosal injectates are used off-label for ESD at this time [20].

To date, there have been a number of trials comparing different submucosal injection fluids for ESD use [21-26]. Ferreira et al. have investigated a number of existing injection solutions in a meta-analysis, including 11 randomized controlled trials (RCTs) with two on ESD. They concluded that sodium hyaluronate and normal saline injectates were clinically equivalent [13]. Recently, Spadaccini et al. published an RCT in a porcine model showing that a new biocompatible polymer SIC-8000 (Eleview) was more effective for ESD and EMR compared to saline solution [27]. Mehta et al. found that lower injection volumes and fewer repeat injections were required with HAES and Eleview in comparison to normal saline for the same mucosal lifting effect [26].

In general, normal saline is reported to be a safe solution with regard to adverse events and cost-effectiveness. In terms of mucosa lifting, it is inferior to more viscous solutions [13]. In an RCT, hydroxyethyl starch has been shown to increase mucosal lifting time and reduce the need for additional injections in comparison to normal saline solution [28].

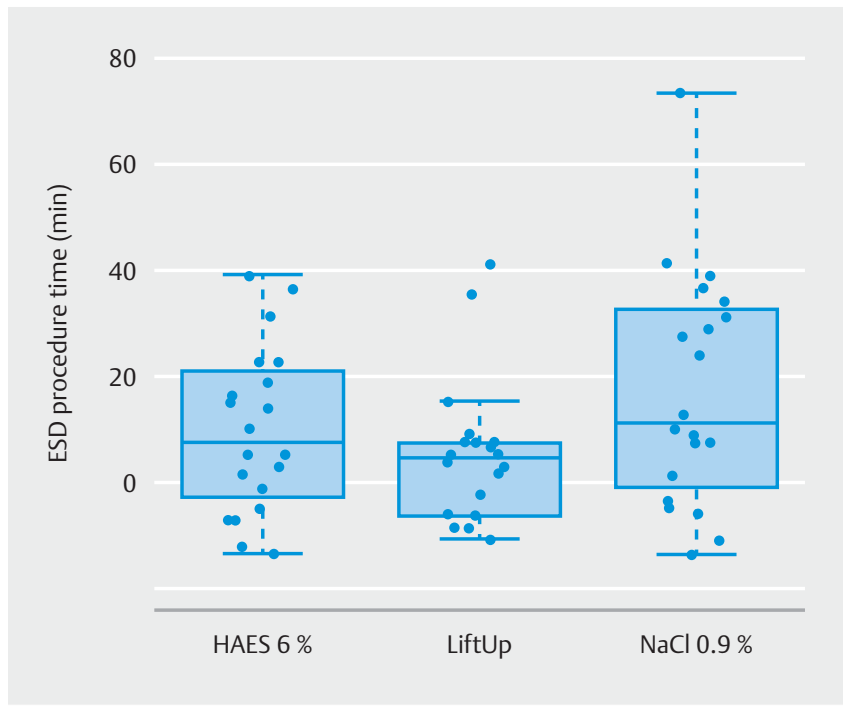

- Fig. 6 ESD procedure time (minutes) dependent on the injection substance.

A newly developed injection material, LiftUp, is currently undergoing investigation for its use in ESD and EMR. In this study, we conducted a prospective, randomized ex vivo study comparing the new fluid with two well-investigated injectates, normal saline solution and HAES. The results of this study demonstrate that LiftUp had significantly better lift quality scores and required significantly less volume injected when compared to HAES and $\mathrm{NaCl}$. LiftUp provided a more long lasting cushion effect, which enabled a secure submucosal dissection with no perforations in the LiftUp group. As a result of its higher viscosity, the injection time for LiftUp was statistically longer than for $\mathrm{NaCl}$ $0.9 \%$ and HAES $6 \%$, but significantly less volume of LiftUp was needed. In the LiftUp group, we performed one injection at the beginning of the procedure and no more repeat injections were required for the remainder of the ESDs. Furthermore, at body temperature, the LiftUp material does not diffuse into the surrounding tissue. In this study, we found that procedure times and complications were not significantly different between groups.

This current study does have some limitations. First, the copolymer was only compared to $\mathrm{HAES} 6 \%$ and $\mathrm{NaCl} 0.9 \%$ and not to all injectates available for ESD. However, to date, HAES 6\% and $\mathrm{NaCl} 0.9 \%$ are the best studied injectates. A further limitation is that the research was performed in an ex vivo model. The effects of blood flow, tissue movement, tissue healing, histopathological evaluation, and other physiological factors which may affect tissue fluid diffusion and durability of a submucosal cushion could not be investigated.

We conclude that LiftUp has similar safety and efficiency qualities compared to $\mathrm{NaCl}$ and $\mathrm{HAES}$, but requires significantly less injected volume and has a preferable subjective quality score to the blinded endoscopist. LiftUp, with its characteristic of a thermally sensitive transition by body temperature, can effectively maintain mucosal elevation, and has ideal lifting prop- 
erties. Further clinical studies are warranted, to analyze its characteristics in human ESD procedures.

\section{Acknowledgments}

The authors would like to thank Song-Sa Dammer (former head nurse, St. Bernward Hospital, Hildesheim) for her services and support during the experiments. Furthermore, we would like to thank Ovesco AG, FujiFilm Europe GmbH and the Department of Gastroenterology, and Associates Dr. Kerzel and Professor Maiss who provided material and technical support. We would also like to thank the Institute of Farm Animal Genetics (FLI Mariensee) for allowing the study to be carried out there.

\section{Competing interests}

Peter Koehler, Jürgen Hochberger, Jürgen Maiss, Sini Milenovic, Mark Gromski, Ulrich Baulain, Carlo Jung and Volker Ellenrieder have no conflicts of interest or financial ties to disclose.

Edris Wedi received material support for research and lecture fees from Ovesco Endoscopy AG. Chi-Nghia Ho and Conrad Gabor declare that they hold management positions with Ovesco Endoscopy AG.

\section{References}

[1] Wang J, Zhang XH, Ge J et al. Endoscopic submucosal dissection vs endoscopic mucosal resection for colorectal tumors: a meta-analysis. World J Gastroenterol 2014; 20: $8282-8287$

[2] Hochberger ], Koehler P, Kruse E et al. Endoscopic submucosal dissection. Der Internist 2013; 54: 287-301

[3] Oka S, Tanaka S, Kaneko I et al. Advantage of endoscopic submucosal dissection compared with EMR for early gastric cancer. Gastrointest Endosc 2006; 64: 877-883

[4] Nakamoto S, Sakai Y, Kasanuki J et al. Indications for the use of endoscopic mucosal resection for early gastric cancer in Japan: a comparative study with endoscopic submucosal dissection. Endoscopy 2009; 41: $746-750$

[5] Tamegai Y, Saito $Y$, Masaki $N$ et al. Endoscopic submucosal dissection: a safe technique for colorectal tumors. Endoscopy 2007; 39: 418 422

[6] Fujishiro M, Yahagi N, Kashimura K et al. Different mixtures of sodium hyaluronate and their ability to create submucosal fluid cushions for endoscopic mucosal resection. Endoscopy 2004; 36: 584-589

[7] Ferlitsch M, Moss A, Hassan C et al. Colorectal polypectomy and endoscopic mucosal resection (EMR): European Society of Gastrointestinal Endoscopy (ESGE) Clinical Guideline. Endoscopy 2017; 49: $270-297$

[8] Rosenberg N. Submucosal saline wheal as safety factor in fulguration or rectal and sigmoidal polypi. AMA Arch Surg 1955; 70: 120-122

[9] Uraoka T, Saito Y, Yamamoto K et al. Submucosal injection solution for gastrointestinal tract endoscopic mucosal resection and endoscopic submucosal dissection. Drug Des Dev Ther 2009; 2: 131-138

[10] Committee AT, Maple JT, Abu Dayyeh BK et al. Endoscopic submucosal dissection. Gastrointest Endosc 2015; 81: 1311-1325

[11] Al-Taie OH, Bauer Y, Dietrich CG et al. Efficacy of submucosal injection of different solutions inclusive blood components on mucosa elevation for endoscopic resection. Clin Exp Gastroenterol 2012; 5: $43-48$

[12] Yamamoto $\mathrm{H}$, Yube $\mathrm{T}$, Isoda $\mathrm{N}$ et al. A novel method of endoscopic mucosal resection using sodium hyaluronate. Gastrointest Endosc 1999; 50: $251-256$

[13] Ferreira AO, Moleiro J, Torres J et al. Solutions for submucosal injection in endoscopic resection: a systematic review and meta-analysis. Endosc Int Open 2016; 4: E1 -E16

[14] Yandrapu H, Desai M, Siddique S et al. Normal saline solution versus other viscous solutions for submucosal injection during endoscopic mucosal resection: a systematic review and meta-analysis. Gastrointest Endosc 2017; 85: 693 -699

[15] Hochberger ], Matthes K, Maiss J et al. Training with the compactEASIE biologic endoscopy simulator significantly improves hemostatic technical skill of gastroenterology fellows: a randomized controlled comparison with clinical endoscopy training alone. Gastrointest Endosc 2005; 61: $204-215$

[16] Schofl R, Buchmeier B, Hauder G. Adaptation of the Erlangen Active Simulator for Interventional Endoscopy (EASIE) model for transmural pancreatic pseudocyst drainage. Endoscopy 2006; 38: 100

[17] Gotoda T. A large endoscopic resection by endoscopic submucosal dissection procedure for early gastric cancer. Clin Gastroenterol Hepatol 2005; 3: S71-73

[18] Hochberger ], Kruse E, Wedi E et al. Training in endoscopic mucosal resection and endoscopic submucosal dissection. In: Cohen J. Successful gastrointestinal endoscopy. Oxford: Wiley-Blackwell; 2011: S204-237

[19] Repici A, Hassan C, De Paula PessoaD et al. Efficacy and safety of endoscopic submucosal dissection for colorectal neoplasia: a systematic review. Endoscopy 2012; 44: 137-150

[20] Pimentel-Nunes P, Dinis-Ribeiro M, Ponchon T et al. Endoscopic submucosal dissection: European Society of Gastrointestinal Endoscopy (ESGE) Guideline. Endoscopy 2015; 47: 829-854

[21] Sumiyama K, Toyoizumi H, Ohya TR et al. A double-blind, block-randomized, placebo-controlled trial to identify the chemical assistance effect of mesna submucosal injection for gastric endoscopic submucosal dissection. Gastrointest Endosc 2014; 79: 756 - 764

[22] Liu W, Zhao M, Liu W et al. A feasibility study of a thermally sensitive elastin-like polypeptide for submucosal injection application in endoscopic resection in 3 animal models. Gastrointest Endosc 2015; 82: $944-952$

[23] Kusano T, Etoh T, Akagi T et al. Evaluation of $0.6 \%$ sodium alginate as a submucosal injection material in endoscopic submucosal dissection for early gastric cancer. Dig Endosc 2014; 26: 638-645

[24] Kim YD, Lee J, Cho JY et al. Efficacy and safety of 0.4 percent sodium hyaluronate for endoscopic submucosal dissection of gastric neoplasms. World J Gastroenterol 2013; 19: 3069-3076

[25] von Renteln D, Dulai PS, Pohl H et al. Endoscopic submucosal dissection with a flexible Maryland dissector: randomized comparison of mesna and saline solution for submucosal injection (with videos). Gastrointest Endosc 2011; 74: 906 - 911

[26] Mehta N, Strong AT, Franco M et al. Optimal injection solution for endoscopic submucosal dissection: a randomized controlled trial of Western solutions in a porcine model. Dig Endosc 2018; 30: 347 - 353

[27] Spadaccini M, Hassan C, Maselli R et al. Efficacy and safety of SIC-8000 (Eleview(R)) for submucosal injection for endoscopic mucosal resection and endoscopic submucosal dissection in an in vivo porcine model. Dig Liver Dis 2018; 50: 260 - 266

[28] Fasoulas K, Lazaraki G, Chatzimavroudis G et al. Endoscopic mucosal resection of giant laterally spreading tumors with submucosal injection of hydroxyethyl starch: comparative study with normal saline solution. Surg Laparosc Endosc Percutan Tech 2012; 22: 272-278 